\title{
Féeries
}

Études sur le conte merveilleuX, XVII ${ }^{e}$ XIXe siècle

9 | 2012

Le dialogisme intertextuel des contes des Grimm

\section{Reconfigurations couleur locale : Le Petit chaperon rouge et Rotkäppchen au Brésil}

Reconfigurations in local color: Le Petit Chaperon rouge and Rotkäppchen in Brasil

\section{Márcio Venício Barbosa}

\section{(2) OpenEdition}

Journals

Édition électronique

URL : http://journals.openedition.org/feeries/823

DOI : $10.4000 /$ feeries. 823

ISSN : $1957-7753$

Éditeur

UGA Éditions/Université Grenoble Alpes

Édition imprimée

Date de publication : 15 octobre 2012

Pagination : 139-160

ISBN : 978-2-84310-233-2

ISSN : 1766-2842

\section{Référence électronique}

Márcio Venício Barbosa, « Reconfigurations couleur locale : Le Petit chaperon rouge et Rotkäppchen au Brésil », Féeries [En ligne], 9 | 2012, mis en ligne le 15 octobre 2013, consulté le 07 septembre 2020. URL : http://journals.openedition.org/feeries/823 ; DOI : https://doi.org/10.4000/feeries.823

\section{(c) Féeries}




\title{
RECONFIGURATIONS COULEUR LOCALE :
}

\author{
LE PETIT CHAPERON ROUGE ET ROTKÄPPCHEN \\ AU BRÉSIL
}

OUT EN POSSÉDANT D'IMMENSES FORETTS, une faune locale spécifique,
et des légendes issues de ses diverses tribus aborigènes, portant sur
les rapports entre l'homme et la nature, le Brésil a pourtant accueilli sans réserve la vieille histoire de cette petite fille qui, au cours de sa traversée d'une forêt d'Europe, fait la rencontre insolite d'un loup. Bien qu'il existe un loup guará sur le territoire brésilien, le loup de l'imaginaire enfantin est un grand loup européen, et c'est lui que chaque enfant qui écoute l'histoire de Perrault ou des Grimm sent sauter sur lui, comme s'il était l'innocente petite fille dans la maison de sa grand-mère.

Ce sentiment traverse les générations depuis le XIX ${ }^{\mathrm{e}}$ siècle, quand commencent à paraître au Brésil des publications destinées aux enfants, comme le remarque Cecília Meireles, poétesse très connue de la deuxième génération moderniste brésilienne, dans son livre consacré aux Problèmes de la littérature de jeunesse :

O século XIX, no Brasil, oferece já um panorama variado de leituras infantis. Mas o mesmo não se pode dizer dos séculos anteriores. A incipiente instrução dos tempos coloniais era impedimento natural ao uso de livros, principalmente dessa espécie. Pelo menos do seu uso generalizado. A leitura não era uma conquista popular.

Mas a Europa, pela mesma época, já possuía livros que só mais tarde viemos a conhecer. Uns, tinham sido escritos especialmente para certos leitores, e depois se divulgaram; outros, foram desde o principio pensados para todas as crianças. Assim, se La Fontaine deu a velha fábulas a forma incomparável do livro destinado ao Delfim de França, os contos de Perrault e os de $M^{m e}$ d'Aulnoy foram recolhidos da tradição popular como quem salva um tesouro para todas as crianças do mundor.

I. C. Meireles, Problemas da literatura infantil, 1984, p. 37-38. 
Le XIX ${ }^{\mathrm{e}}$ siècle, au Brésil, offre déjà un vaste panorama de lectures pour enfants. Mais on ne peut pas en dire autant des siècles précédents. Le niveau d'instruction encore très bas pendant l'ère coloniale constituait un obstacle naturel à l'usage des livres, surtout de ce domaine - du moins à son usage par tous. La lecture n'était pas une conquête populaire.

Mais l'Europe, à la même époque, avait déjà les livres qui sont venus peu de temps plus tard à notre connaissance. Les uns ont été écrits spécialement pour certains lecteurs particuliers et ont par la suite été diffusés, d'autres ont été dès l'origine conçus pour tous les enfants. C'est ainsi que La Fontaine a donné à de vieilles fables la forme incomparable du livre destiné au Dauphin de France, et que les contes de Perrault et ceux de $\mathrm{M}^{\mathrm{me}} \mathrm{d}$ 'Aulnoy ont été recueillis dans la tradition populaire comme s'il s'agissait d'un trésor à sauvegarder pour tous les enfants du monde ${ }^{2}$.

Je ne discuterai pas ici la pertinence du classement de contes tels que Le Petit Chaperon Rouge dans la catégorie de la littérature de jeunesse. Les réflexions de Ute Heidmann ${ }^{3}$ à ce propos montrent bien que les mouvements dialogiques présidant à leur composition exigent un lecteur beaucoup plus expérimenté que ne l'est un enfant. Ce même dialogisme, comme l'ont montré Heidmann et Adam ${ }^{4}$, n’autorise pas à affirmer de but en blanc, loin s'en faut, que ces contes sont de simples adaptations de contes populaires, ainsi que le prétend Meireles.

Toute analyse des contes est inévitablement confrontée au fait que des siècles de commentaires et de rééditions pèsent sur eux. En nous devenant trop familiers par les livres illustrés, le cinéma, les dessins animés et les enregistrements audio, ils ont perdu la distance historique qui correspond à ce qui en a fait des événements de discours au moment de leur apparition. En revenant à la textualité des contes, nous proposons de les (re)lire aussi près que possible de leur état premier de parution'.

Du fait de cette «naturalisation» des contes dans les différentes cultures, leur contextualisation et ce que les auteurs cités précédemment appellent les "événements de discours au moment de leur apparition"sont, bien évidemment, beaucoup plus difficiles à repérer pour un lecteur moderne quand il s'agit des contes classiques. La critique elle-même se soumet très facilement aux grilles d'analyse d'une prétendue tradition orale, en ignorant presque tout du dialogue intertextuel qui alimente la reconfiguration continue des contes. Dans son propos, Cecília Meireles met en avant des

2. Toutes les traductions des citations en portugais sont miennes.

3. Ute Heidmann développe ce thème dans différents articles et livres, mais je renvoie particulièrement à U. Heidmann, "Expérimentation générique et dialogisme intertextuel : Perrault, La Fontaine, Apulée, Straparola, Basile», 201I, p. 45-69.

4. U. Heidmann et J.-M. Adam, Textualité et intertextualité des contes, 2010.

5. Ibid., p. 26. 
auteurs français, ce qui s'explique par la grande présence française au Brésil au XIX ${ }^{e}$ siècle, mais elle n'identifie pas, dans la suite de son livre, les rapports entre les textes brésiliens et les textes européens. Pourtant, tout au long du $\mathrm{Xx}^{\mathrm{e}}$ siècle, nous avons déjà au Brésil des productions qui vont un peu au-delà de la simple traduction des contes et, même si Perrault, également cité par Meireles, est connu dans ce pays et toujours mentionné, surtout dans les études portant sur la littérature de jeunesse, ce n'est pas son Petit Chaperon Rouge qui dominera la production des reconfigurations brésiliennes. Le Chapeuzinho vermelho brésilien est plutôt celui du texte des frères Grimm, Rotkäppchen, qui occupe l'avant-scène et qui est reproduit, réécrit, adapté, transposé dans plusieurs reconfigurations.

Il n'y a pas d'études qui expliquent ce choix au Brésil, mais on peut très facilement penser que la fin heureuse, quoique fantastique (ou justement parce que fantastique) du conte des Grimm plait beaucoup plus aux enfants et à leurs parents. En tout cas, c'est le texte que l'on trouve le plus fréquemment reproduit dans les anthologies scolaires et utilisé dans les adaptations pour enfants.

Je ne me livrerai pas ici à un relevé exhaustif de la présence de ce conte dans la littérature brésilienne, ni à l'analyse de ses diverses traductions en brésilien ${ }^{6}$. J'ai choisi de parler de quatre réécritures qui ont un caractère dialogique plus marqué, permettant une recontextualisation et offrant une réponse au conte européen, avec un humour caractéristique d'une certaine irrévérence brésilienne pour les trois premières, qui sont plutôt liées au conte des Grimm. Suivant toujours ce même principe dialogique, la dernière réécriture, porteuse d'un sens tragique, est plutôt associée, quant à elle, au conte de Perrault.

Avant de parler des trois premières réécritures de Rotkäppchen, il faut mentionner son passage par le «Sitio do Pica-pau Amarelo ${ }^{7}$, nom donné par Monteiro Lobato à un univers enfantin construit autour d'une grandmère sympathique et accueillante, Dona Benta. Dans les livres qui mettent en place cet univers, de nombreux personnages mythologiques, féeriques et

6. Pour la traduction des contes de Grimm au Brésil, consulter le site de Karin Volobuef, qui propose un tableau des traductions les plus connues, étant donné que Kinder-und Hausmärchen n’a jamais été publié intégralement en traduction brésilienne. Pour les contes de Perrault, on peut citer les traductions de Maria Luiza Borges (Perrault, Contos de Fadas, 20Io) et de Mario Laranjeira (Perrault, Contos e fábulas, 2007).

7. On pourrait traduire en français «Sítio do Pica-pau Amarelo» par «La ferme du Pivert jaune», «sítio» étant la désignation d'une propriété rurale de petite taille. Le livre de Monteiro Lobato, O Pica-pau Amarelo a été publié en 1939, mais depuis I920 l'auteur avait déjà publié une trentaine de livres d'histoires ayant pour cadre cet univers. 
même historiques rendent visite au «sitio» et participent aux histoires de Pedrinho et Narizinho, les deux petits enfants de Dona Benta qui viennent régulièrement y faire de longs séjours et aiment écouter les histoires de leur grand-maman. Ils sont toujours accompagnés d'une impertinente poupée en tissu, Emília, et d'un sage Vicomte, fabriqué à partir d'un épi de maïs. Dans l'un de ces livres, Pedrinho, Narizinho et Emília visitent le château de Blanche-Neige en compagnie de Peter Pan et ils parlent du merveilleux. C'est alors qu'ils prennent pour exemple le cas du Petit Chaperon Rouge, appelée dans ce livre "Capinha Vermelha» (le petit chaperon s'étant changé en petite cape) :

- Que coisa curiosa! - disse Narizinho. No Mundo da Fábula ninguém morre duma vez. Peter já venceu esse Gancho e o fez afogar-se no mar e ser engolido pelo jacaré - e depois disso o Capitão já nos apareceu lá em casa e agora vai aparecer novamente aqui...

- Se não fosse assim - explicou Branca - isso não seria nenhum Pais-das-Maravilhas. $O$ maravilhoso está justamente nisso...

- Foi também o que aconteceu para o lobo que devorou a avó de Capinha. Morreu a machadadas, e no entanto, continua a viver e a farejar avós - como naquele dia lá no sítio ${ }^{8}$.

- Comme c'est curieux! - dit Narizinho. Dans le Monde de la Fable personne ne meurt définitivement. Peter a déjà vaincu ce Capitaine Crochet et l'a fait se noyer dans la mer et avaler par un caïman - et après ça le Capitaine est déjà revenu chez nous et il va encore venir ici...

- Si ce n'était pas ainsi - explique Blanche-Neige - il ne s'agirait pas d'un Pays des Merveilles. Le merveilleux est justement là...

- C'est aussi ce qui s'est passé avec le loup qui a avalé la grand-mère de la Petite Cape. Il a été tué à coups de hache et pourtant il est toujours vivant et il renifle les mémés comme ce jour-là au «sítio».

Monteiro Lobato explique alors à ses jeunes lecteurs le fonctionnement du Pays des Merveilles, passage quelque peu didactique dans un livre lu et relu par plusieurs générations de jeunes Brésiliens. Les enfants découvrent, à partir de ce dialogue, le caractère éternel des personnages des contes, même les plus cruels, voire même pervers, c'est-à-dire profondément malfaisants et enclins au mal, et cette découverte sera déterminante pour leur imaginaire fantastique : si le loup peut venir au "sitio" même après avoir été tué, il peut donc aussi aller n’importe où. L'idée qui fonde ce type de raisonnement s'ancre dans le conte des Grimm. Dans celui de Perrault, le loup reste toujours vivant et la «résurrection perpétuelle» serait donc l'affaire du Petit Chaperon Rouge et de sa grand-mère. Quoi qu'il en soit, l'important est de faire valoir, dans un livre fondateur de la littérature de

8. Monteiro Lobato, O Picapau amarelo, I971, p. 55-56. 
jeunesse brésilienne, le choix effectué par Monteiro Lobato du texte des Grimm, qu'il avait déjà traduit et publié dans deux petites anthologies?.

Il ne s'agit pas d'ailleurs d'un choix complètement innocent ni même basé uniquement sur la facilité dont nous parlions plus haut à propos de la fin «heureuse» de Rotkäppchen. Si elle l'est en effet pour les personnages humains du conte, en revanche pour le loup elle est tout à fait catastrophique. Mais la possibilité de cette "résurrection perpétuelle» vient remplacer très efficacement la moralité lisible chez Perrault. Il ne s'agit plus de se méfier des «loups doucereux», selon son conseil de Perrault aux jeunes filles : d'après les Grimm et conformément à la définition du merveilleux présente chez Monteiro Lobato, tout enfant doit avoir peur du loup lui-même, car il peut revenir n'importe quand. Et les mères brésiliennes ont toujours su trouver les occasions de s'en servir à leur gré.

\section{La reconfiguration discursive dans Chapeuzinho Vermelho, de Millôr Fernandes}

L'histoire du Chaperon Rouge, configurée comme un conte d'effroi chez Perrault ou d'avertissement chez les Grimm, incarne donc la peur dans l'imaginaire brésilien, et pas seulement l'imaginaire enfantin. Longtemps cette histoire a circulé sous la forme d'une simple traduction, du conte de Grimm le plus souvent. Une seule exception peut être enregistrée dans l'anthologie présentée par Ana Maria Machado ${ }^{10}$ qui reproduit les deux contes, Le Petit Chaperon Rouge et Rotkäppchen.

À partir des années 1960, on voit pourtant paraittre quelques réécritures différentes, qui opèrent une vraie recontextualisation de cette histoire. Je citerai d'abord celle due à Millôr Fernandes, écrivain brésilien, humoriste, dessinateur, auteur de théâtre, récemment disparu. Millôr s'est rendu très célèbre dans les années 1960 et 1970 par ses apparitions fréquentes dans les journaux et revues brésiliens, dont le fameux Pasquin, l'un des seuls à s'opposer au régime militaire, en toute irrévérence. Sa réécriture du Chaperon Rouge, publiée en 1967, attaque les analyses universitaires. Il ponctue chaque situation du conto ${ }^{\text {II }}$ d'une explication pseudo-scientifique, comme on peut le voir dans le dialogue suivant entre la petite fille et le loup, dans le bois :

9. J. et W. Grimm, Contos de Grimm, 1969 et Novos contos de Grimm, I969 (trad. de Monteiro Lobato).

Io. A. M. Machado, Contos de fadas de Perrault, Grimm, Anderson e outros, Jorge Zahar, 20 Io.

II. La dénomination générique sera utilisée en portugais, langue originale des textes étudiés dans cet article, «afin de souligner, selon Ute Heidmann, les différences culturelles et historiques et afin 
Ouvindo isso o Lobo saiu correndo, estimulado por desejos reprimidos (Freud: "Psychopathology of Everiday Life", The Modern Library Inc. N.Y.). Chegando na casa da avozinha ele engoliu-a de uma vez - o que, segundo o conceito materialista de Marx indica uma intenção crítica do autor, estando oculta ai a idéia do capitalismo devorando o proletariado-e ficou esperando, deitado na cama, fantasiado com a roupa da avón ${ }^{12}$.

Après avoir écouté la petite fille, le Loup se met à courir, sous la pulsion de désirs refoulés (Freud : "Psychopathologie de la vie quotidienne», The Modern Library Inc. N.Y.). Une fois arrivé à la maison de la grand-maman, il n'en fait qu'une bouchée - ce qui, selon la conception matérialiste de Marx, dénote une intention critique de l'auteur, l'idée du capitalisme qui dévore le prolétariat étant ici sous-jacente - et il attend, allongé sur le lit, déguisé avec les vêtements de la grand-mère.

Alors qu'en arrivant chez sa grand-mère, Rotkäppchen «s'étonna de trouver la porte ouverte et, en entrant dans la pièce, tout lui sembla étrange ${ }^{\mathrm{r3}}$ ", le Chaperon Rouge de Millôr Fernandes ne soupçonne pas le danger et à cela aussi une explication scientifique est apportée :

Chapeuzinho Vermelho chegou e não percebeu que o lobo não era sua avó, porque sofria de astigmatismo convergente, que é uma perturbação visual oriunda da curvatura da córnea. Nem percebeu que a voz não era a da avó, porque sofria de Otite, inflamação do ouvido, nem reconheceu nas suas palavras, palavras cheias de má-fé masculina, porque afinal, eis o que ela era mesmo: esquizofrênica, débil mental e paranóica pequenas doenças que dão no cérebro, parte-súpero-anterior do encéfalo. (A tentativa muito comum da mulher ignorar a transformação do Homem é profusamente estudada por Kinsey em "Sexual Behavior in the Human Female", W. B. Saunders Company, Publishers. $)^{14}$

Le Petit Chaperon Rouge est arrivé et ne s'est pas rendu compte que le loup n'était pas sa grand-mère, à cause de son astigmatisme convergent, une perturbation visuelle de la courbure de la cornée. Elle ne s'est même pas aperçue que la voix entendue n'était pas celle de sa grand-mère, car elle souffrait d'une otite, inflammation de l'oreille, et n'a même pas reconnu dans ses paroles, des paroles pleines de mauvaise foi masculine, car, en fin de compte, voilà ce qu'elle était en vérité : schizophrène, débile et paranoïaque, petites maladies qui se manifestent dans le cerveau, dans la partie supéro-antérieure de l'encéphale. (La tentative très répandue chez la femme d'ignorer la transformation de l'Homme est amplement étudiée par Kinsley dans «Le comportement sexuel de la femme», W. B. Sauniers Company, Publishers.)

Dans cet extrait se font sentir les échos du discours d'émancipation féminine, chargé d'exemples scientifiques et s'appuyant sur des arguments d'autorité, bien nécessaires pour parvenir à la libération de la femme. C'est

d'éviter d'induire l'illusion de l'universalité d'un genre conte». (U. Heidmann et J.-M. Adam, Textualité et intertextualité des contes, 2010, p. 27.)

I2. M. Fernandes, Liçôes de um ignorante, 1967, p. 3I.

I3. J. et W. Grimm, Contes pour les enfants et la maison, Paris, José Corti, 2009, p. 165.

I4. M. Fernandes, ouvr. cité, 1967, p. 31. 
ce qui explique les adjectifs associés à la jeune fille «schizophrène, débile et paranoïaque», seule issue possible pour une femme qui se laisse piéger comme le Petit Chaperon Rouge. Ce discours pseudo-scientifique produit un effet humoristique complexe chez le lecteur moyen, car il s'agit d'un discours difficile d'accès pour le grand public. Et c'est justement cet aspect que Millôr Fernandes attaque dans sa reconfiguration. Il transforme une histoire largement diffusée dans la culture brésilienne et sa reconfiguration discursive est une fois encore ancrée dans le texte des Grimm :

Mas, para salvação de Chapeuzinho Vermelho, apareceram os lenhadores, mataram cuidadosamente o Lobo, depois de verificar a localização da avó através da Roentgenfotografia. E Chapeuzinho Vermelho viveu tranqüila 57 anos, que é a média da vida humana segundo Maltus, Thomas Robert, economista inglês nascido em 1766 , em Rookew, pequena propriedade de seu pai, que foi grande amigo de Rousseau ${ }^{\text {I5 }}$.

Mais, pour le salut du Petit Chaperon Rouge, les bûcherons sont venus et ils se sont appliqués à tuer le Loup, après avoir vérifié par radiographie la localisation exacte de la grand-mère. Et le Petit Chaperon Rouge a vécu tranquille jusqu’à 57 ans, ce qui est la moyenne de vie humaine, selon Maltus, Thomas Robert, économiste anglais né en 1766 , à Rookew, petite propriété de son père, qui a été très ami avec Rousseau.

Comme dans Rotkäppchen, la petite fille du conto de Millôr Fernandes est sauvée du ventre du Loup, mais elle ne vivra pas éternellement, chose interdite aux humains surtout dans le cadre contextuel d'un discours scientifique. La science, qui alors le vent en poupe, conquiert une place de choix dans la vie sociale, et tous les événements quotidiens trouvent une explication scientifique assez convaincante : c'est ce phénomène qui est ici parodié.

\section{La grand-mère "branchée", dans Chapeuzinho vermelho de raiva, de Mario Prata}

Le conto de Mario Prata présente à Rotkäppchen une réponse bien différente de la parodie de discours scientifique à laquelle s'est livré Millôr Fernandes. Elle est en effet construite dans un registre complètement prosaïque. Mario Prata, auteur de romans et de pièces de théâtre, écrit aussi pour la télévision au Brésil et au Portugal. Il reconfigure la vieille histoire dans un texte intitulé Chapeuzinho vermelho de raiva (Petit chaperon rouge de rage), histoire qui se cantonne au dialogue entre La grand-mère loup et le Petit Chaperon Rouge. Celui-ci donne des réponses contextualisées dans la vie contemporaine et fait toujours des observations très ordinaires :

I5. Ibid. 
- Mas vovó, que olho vermelho... E grandão... Queque houve?

- Ah, minha netinha, estes olhos estão assim de tanto olhar para você. Aliás, está queimada, hein?

- Guarujá, vovó. Passei o fim de semana lá. A senhora não me leva a mal, não, mas a senhora está com um nariz tão grande, mas tão grande! Tá tão esquisito, vovó.

- Ora, Chapéu, é a poluição. Desde que começou a industrialização do bosque que é um Deus nos acuda. Fico o dia todo respirando este ar horrivel. Chegue mais perto, minha netinha, chegue ${ }^{16}$.

— Mais mémé, ce qu'ils sont rouges vos yeux... Et grands... Qu'est-ce qui s'est passé? - Ah, ma petite, ces yeux sont comme ça à force de t'avoir regardée tant de fois. D'ailleurs, t'es bien bronzée, hein?

— Guarujáa ${ }^{17}$, mémé. J’y ai passé le week-end. Le prenez pas mal, mais faut que je vous dise que vous avez un nez si grand, mais si grand! Il est trop bizarre, mémé.

-Écoute, Chapeau, c'est la pollution. Depuis qu'on a commencé l'industrialisation du bois, c'est Sauve-qui-peut. Je passe la journée à respirer cet air horrible. Approche-toi, ma petite, viens.

La grand-mère change le surnom de la petite fille et au lieu de l'appeler «Petit Chaperon», elle dit tout simplement "Chapéu» (en français "Chapeau»), manière de parler caractéristique de la jeunesse des années I970 au Brésil, qui consistait à créer des surnoms suivant un principe de réduction ou d'économie verbale.

Dans le panier, le «Chapeu» de Mário Prata ne transporte plus de beurre, comme chez Perrault, mais des produits industrialisés, qui ont commencé à circuler dans le marché brésilien à cette époque, après l'ouverture commerciale du pays aux investisseurs étrangers et l'implantation au Brésil de grandes usines multinationales. "Chapéu» se rend compte qu'elle a failli oublier de donner le panier à sa grand-mère :

- Puxa, já ia me esquecendo: a mamãe mandou umas coisas para a senhora. Olha ai: margarina, Helmmans, Danone de frutas e até uns pacotinhos de Knorr, mas é para a senhora comer um só por dia, viu? Lembra da indigestão do carnaval ${ }^{18}$ ?

— Ah là la! j'allais oublier : maman vous a envoyé des choses. Regardez moi ça : de la margarine, de la mayonnaise Helmmans, du yaourt Danone et même des petits paquets de sauce Knorr, mais vous devez en manger seulement un seul par jour, compris? Vous vous souvenez de l'indigestion du carnaval?

À la fin du dialogue, Chapeau, comme l'appelle la grand-mère/loup, fait des observations sur la coiffure de son aïeule, qui sont suivies d'une fin insolite :

16. M. Prata, Chapeuzinho vermelho de raiva, 1970.

I7. N. D. T. : Guarujá est le nom d'une ville et d'une plage à côté de São Paulo.

I8. M. Prata, ouvr. cité, I970. 
- Por falar em juventude o cabelo da senhora está um barato, hein? Todo desfiado, pra cima, encaracolado. Que qué isso?

- Também tenho que entrar na moda, não é, minha filha? Ou você queria que eu fosse domingo ao programa do Chacrinha de coque e com vestido preto com bolinhas brancas? Chapeuzinho pula para trás:

- E esta boca imensa???!!!

A avó pula da cama e coloca as mãos na cintura, brava:

- Escuta aqui, queridinha: você veio aqui hoje para me criticar é $e^{19} ? !$

- À propos de jeunesse, vos cheveux sont super, hein? Complètement explosés, hérissés, bouclés. Dis donc!

- Moi aussi, je dois suivre la mode, pas vrai, ma petite? Tu veux que j'aille à l'émission de Chacrinha habillée d'un chiffon et d'une robe noire à pois blancs?

Le Petit Chaperon Rouge fait un bond en arrière :

— Et cette bouche immense???!!!

La grand-mère saute du lit et met les mains sur les hanches, furieuse :

— Écoute, chérie, t'es venue aujourd'hui que pour me faire des critiques?!

Dans ce dialogue les références aux années I970 sont complétées par la mention de Chacrinha, surnom d'un présentateur de télévision brésilien qui a animé une émission hebdomadaire très longtemps, dans une ambiance complètement anarchique, mêlant tous les éléments de la culture brésilienne. Mais il faut surtout noter les préoccupations futiles du loup, qui veut avoir l'air «branché» et qui supporte mal les critiques. Dans cette réécriture, à part le dialogue et les observations de la petite fille sur les mêmes parties du corps de sa grand-mère, on ne retrouve ni l'avertissement de Perrault, ni la peur de la rencontre avec un loup féroce. Au contraire, le loup possède plutôt les traits d'une mondanité surprenante. Le seul rapprochement possible avec le conte de Perrault, si l'on s'en tient à la leçon délivrée par sa moralité, réside dans le retournement de la lecture analogique. Ce ne sont plus les amants "doucereux» qui sont assimilés au loup prédateur, mais c'est le loup lui-même, travesti en grand-mère, qui apparaît comme un personnage soucieux de paraître jeune et "dans le vent», comme on disait dans les années I970.

\section{La méthode poétique dans Chapeuzinho Amarelo, de Chico Buarque}

Si la peur n'est pas présente dans le texte de Mario Prata, dans celui de Chico Buarque elle occupe tout le devant de la scène. Chico Buarque, chanteur, compositeur et écrivain, très connu et estimé au Brésil, propose, en 1979,

I9. M. Prata, ouvr. cité, I970. 
un Chaperon jaune, couleur associée à la peur constitutive du personnage créé dans ce poème : une petite fille que tout effraie. Avec cet exemple, on quitte le genre du conto brésilien et on se trouve en présence d'un livrepoème à destination des enfants. Chico Buarque l'a d'ailleurs dédié à ses filles et à d'autres enfants de son entourage :

A historinha foi feita para Luisa.

O livro é dela, da Silvia,

da Helena, da Janaina, da Alaíde,

da Luiza, do Antônio e dos outros ${ }^{20}$.

La petite histoire a été faite pour Luísa.

Le livre est à elle, à Silvia,

à Helena, à Janaína, à Alaíde

à Luíza, à Antônio et aux autres.

Parmi tous les exemples de reconfiguration des contes européens présentés dans cet article, celui de Chico Buarque est le seul à être très clairement écrit pour un jeune public. Les autres, malgré la recréation d'un personnage célèbre et très présent dans l'imaginaire enfantin, introduisent des éléments qui les éloignent de ce public, et auxquels ils ne peuvent avoir accès sans accompagnement. Dès le titre du poème, on voit déjà s'opérer la modification apportée dans cette réponse à l'histoire européenne. Ce changement de couleur du Petit Chaperon attire l'attention du jeune lecteur, très habitué au « rouge» traditionnel. Et il posera des questions, bien évidemment, à propos de ce changement. C'est pourquoi on découvre dans la présentation même du personnage, que Chico Buarque joue avec l'expression très populaire au Brésil, "être jaune de peur", liée à l'identification de ce sentiment chez autrui. Par dérivation , on peut même, au Brésil, utiliser le verbe "jaunir» (amarelar), dans des situations où une décision se prend sous son emprise. L'auteur fournit d'emblée la liste de toutes les peurs de son "Chaperon Jaune», qui peuvent bien s'appliquer à tous les enfants :

Tinha medo de trovão.

Minhoca, pra ela, era cobra.

E nunca apanhava sol

porque tinha medo da sombra.

Não ia pra fora pra não se sujar.

Não tomava sopa pra náo ensopar.

Não tomava banho pra náo descolar.

Náo falava nada pra náo engasgar.

Náo ficava em pé com medo de cair.

20. C. Buarque, Chapeuzinho Amarelo, 2005, p. 3.

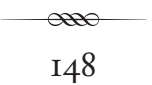


Então vivia parada, deitada, mas sem dormir, com medo de pesadelo ${ }^{21}$.

Elle avait peur du tonnerre.

Un vers de terre c'était pour elle un cobra.

Et elle ne prenait jamais de soleil

car elle avait peur de l'ombre.

Elle n'allait pas dehors pour ne pas se salir.

Elle ne prenait pas de soupe pour ne pas se tremper.

Elle ne prenait pas de douche pour ne pas se décoller.

Elle ne disait pas un mot pour ne pas s'étouffer.

Elle ne se mettait pas debout de peur de tomber.

Alors, elle vivait immobile, couchée, mais sans dormir,

de peur des cauchemars.

On voit bien, à la lecture de ces vers, que les peurs du Petit Chaperon Jaune n'ont pas qu'un seul objet. Elle a littéralement peur de tout. Mais la pire des peurs pour elle, c'est celle du loup. Un loup qui habite en Allemagne, mention qui, dans le livre, a deux fonctions : d'abord, par cette localisation géographique lointaine, l'auteur montre que cette peur n'a pas de raison d'être et qu' elle est plutôt le fruit de l'imagination de la petite fille; ensuite, l'indication explicite du pays des Grimm montre de quel loup il s'agit. Le loup qui hante le Petit Chaperon jaune est semblable à celui qui avait dévoré son homologue rouge européen.

À part cette mention explicite au pays des Grimm, aucune autre caractéristique générique ne renvoie aux contes européens. Le nom du personnage (quoique de couleur différente) et la présence d'un loup produisent certes une association immédiate avec Rotkäppchen, mais ici plus de grand-mère, ni de chasseurs. À vrai dire, il n'y a même plus de loup, puisque c'est la petite fille angoissée qui l'imagine : le loup n'est qu'un fantasme, qu'une terreur intime.

Comme dans le monde du merveilleux tout peut arriver, le Petit Chaperon Jaune rencontre le loup, et il est bien à la hauteur de ses craintes :

E Chapeuzinho Amarelo, de tanto pensar no $L O B O$,

de tanto sonhar com $\angle O B O$,

de tanto esperar o $L O B O$,

um dia topou com ele

que era assim:

2I. C. Buarque, ouvr. cité, 2005 , p. 7. 
carão de $\angle O B O$,

olhão de $\angle O B O$,

jeitão de $L O B O$

e principalmente um bocão

tão grande que era capaz

de comer duas avós,

um caçador,

rei, princesa,

sete panelas de arroz

e um chapéu

de sobremesa ${ }^{22}$.

Et le Petit Chaperon Jaune,

à force de tant penser au LOUP,

à force de tant rêver du LOUP,

à force de tant attendre le LOUP,

un jour est tombée sur lui

qui était ainsi :

grande tête de LOUP,

grands yeux de LOUP,

grand air de LOUP

et, surtout, une gueule

tellement énorme qu'elle était capable

de manger deux grand-mères,

un bûcheron,

un roi, une princesse,

sept casseroles de riz

et un chapeau

comme dessert.

Cet extrait réinvente le dialogue entre la petite fille et le loup, car, étant donné que le Petit Chaperon Jaune «ne disait pas un mot pour ne pas s'étouffer ", la voix poétique doit prendre en charge toute la chronique de la rencontre. Le lecteur peut y trouver énumérées les parties du corps de l'animal, comme dans les textes européens. Tout le potentiel dévorateur du loup est souligné, puisqu'il est capable de consommer non seulement une, mais deux grand-mères, entre autres plats de son vaste menu, et même un "chapeau comme dessert», allusion métonymique servant à introduire la petite fille dans la liste des gourmandises.

Devant le loup, le Petit Chaperon Jaune se trouve face à face avec sa grande peur et elle découvre progressivement que ce qui la provoquait, c'était la peur elle-même :

22. Ibid., p. I5. 
Mas o engraçado é que, assim que encontrou o $L O B O$,

a Chapeuzinho Amarelo

foi perdendo aquele medo,

o medo do medo do medo

de um dia encontrar um LOBO.

Foi passando aquele medo

do medo que tinha do $L O B O$.

Foi ficando só com um pouco

de medo daquele lobo ${ }^{23}$.

Mais ce qui est drôle c'est que,

juste après avoir rencontré le LOUP,

le Petit Chaperon Jaune

a commencé à oublier cette peur,

la peur de la peur de la peur

d'un jour rencontrer le LOUP.

Elle s'en allait, cette peur

de la peur qu'elle avait du LOUP.

Il ne restait qu'un tout petit bout

de peur de ce loup-là.

Le poète utilise les ressources du langage pour montrer la déconstruction de la peur. Par l'effet d'une répétition qui met cette peur en abyme («la peur de la peur de la peur»), son objet perd son importance et, bien évidemment, ses majuscules. Au dernier vers du couplet, "le LOUP» est devenu "ce loup-là", un loup comme les autres et qui ne fait plus peur à la petite fille.

La fin du poème est également malheureuse pour le loup et Chico Buarque trouve une solution linguistique qui permet à la petite fille d'inverser le nom du loup et, ce faisant, de renverser à la fois le sujet et l'objet de la peur : c'est le loup qui commence à la craindre alors. Elle peut dès lors combattre toutes ses peurs passées en recourant à la même méthode :

E o lobo parado assim

do jeito que o lobo estava

já não era mais um $L O-B O$

Era um BO-LO.

Um bolo de lobo fofo,

tremendo que nem pudim,

com medo da Chapeuzim.

23. Ibid., p. I6. 
Com medo de ser comido

com vela e tudo, inteirim. ${ }^{24}$

Et le loup immobile comme ça

tel que le loup était

n'était plus un LO-BO [loup]

Il était un BO-LO [gâteau].

Un gâteau de loup moelleux,

qui tremblait comme un pudding,

de peur du Petit Chapeau.

De peur d'être mangé

avec les bougies et tout, complètement.

En inversant les syllabes du mot LOBO, loup en portugais, on obtient le mot BOLO, gâteau en portugais. La petite héroïne du poème utilise ce même stratagème langagier et poétique qui consiste à jouer avec les signes linguistiques pour faire face à toutes ses autres peurs. Cette méthode produit le même effet qu'une moralité, étant donné qu'elle offre un enseignement aux lecteurs, qui peuvent eux aussi essayer de maîtriser leurs peurs. De cette façon, le poème de Chico Buarque ne répond pas uniquement aux Grimm ou à Perrault, il propose une reconfiguration générique qui communique indirectement avec les deux contes, au moyen de procédés poétiques doublement dialogiques : en reconfigurant la vieille histoire de la petite fille et du loup, il répond à la tradition et, en même temps, il s'adresse aux enfants contemporains, en leur proposant une méthode pour combattre la peur.

\section{$\mathrm{La}$ «nouvelle vieille histoire» : Fita verde no cabelo, de Guimarães Rosa}

Si Chico Buarque apprend aux lecteurs comment se débarrasser du sentiment de peur, le dernier conto que nous verrons ici fait exactement le contraire : c'est la peur qui s'impose dans le conto de João Guimarães Rosa, Fita verde no cabelo - nova velha estória, titre qui, en français, pourrait être traduit par «Ruban vert sur les cheveux». Le sous-titre "nouvelle vieille histoire», indique déjà qu'un dialogue est établi avec d'autres textes, puisqu'il propose toute de suite de raconter une histoire paradoxalement nouvelle et vieille à la fois. Le mot utilisé pour dire «histoire» en portugais, comme souvent chez Guimarães Rosa, est "estória» et non "história». Ce faisant, l'auteur entend mettre en évidence la distinction entre fiction (estória) et science

24. Ibid., p. 25 . 
historique (história). De nos jours, les dictionnaires conseillent l'utilisation de la seule forme "história» dans les deux sens.

Cet écrivain brésilien est considéré comme l'un des plus grands écrivains de langue portugaise. Guimarães Rosa a été aussi diplomate, il a beaucoup voyagé et a toujours été très ouvert aux cultures du monde. Médecin de formation, il s'est toujours montré très attentif aux langues : il maîtrisait le français, l'anglais, l'espagnol, l'allemand et pouvait lire directement l'original des œuvres en grec, en arabe, en latin et en une trentaine d'autres langues. Ainsi commente-t-il son rapport à elles :

Aprendi algumas linguas estrangeiras apenas para enriquecer a minha própria e porque há demasiadas coisas intraduziveis, pensadas em sonhos, intuitivas, cujo verdadeiro significado só pode ser encontrado no som original. ${ }^{25}$

J'ai appris quelques langues étrangères uniquement dans le but d'enrichir la mienne et parce qu'il y a plusieurs choses intraduisibles, pensées pendant les rêves, intuitives, dont la vraie signification ne peut être trouvée que dans le son original.

Cette proximité des langues étrangères traverse ses contos et ses romans, qui créent un univers perdu à l'intérieur du Brésil : le sertão («l'arrière-pays»), qui possède sa langue propre, construite à partir des formes d'expression des habitants du nord de Minas Gerais, mais pétrie de références savantes et d'apports étrangers. À côté des thèmes associés au sertão, Rosa s'est penché plus d'une fois sur les problèmes de l'enfance, en créant des personnages pleins d'humanité et d'émotion, comme Miguilim. Une étude des personnages-enfants de Rosa pourrait très bien orienter la lecture du conto dont je parlerai ici. Pour résumer, on peut dire que tous les enfants de son œuvre - et il y en a beaucoup - se trouvent à un certain moment confrontés à un grand événement, qui bouleverse définitivement leur vie, tel un rite de passage après lequel l'enfance a disparu. C'est ce qui arrive à la petite fille de "Fita verde no cabelo» ("Ruban vert dans les cheveux»)

Une petite fille quitte le village, lieu plutôt calme dans lequel les vieilles personnes s'appliquent à vieillir, les hommes et les femmes à attendre, les garçons et filles à naître puis à grandir. L'auteur la présente comme celle qui n'est pas encore suffisamment sage. Pour la décrire, il utilise une expression syntaxiquement incomplète : elle est "celle qui pour le moment» ("Aquela que por enquanto »). Cette phrase interrompue crée dès le premier paragraphe une attente chez le lecteur, qui sait alors que quelque chose va arriver à cette petite fille.

25. G. Lorenz, «Diálogo com Guimarães Rosa», 1991, p. 87. 
Comme dans les contes européens, sa mère l'envoie chez sa grand-mère munie d'un panier et d'un pot. Dans le deuxième paragraphe, et non avant, on retrouve l'expression figée qui ouvre en général les contes, mais modifiée par l'auteur : «Tout était une fois» ("Tudo era uma vez»). Le déplacement de cet habituel incipit opère des changements dans la conception du temps mythique propre au genre. Il n'est pas établi d'emblée, car le conto s'ouvre sur cette déclaration : "Il y avait un village» ("Havia uma aldeia»). Placée au deuxième paragraphe, la formule figée produit l'effet d'un adjectif caractérisant l'ambiance dans laquelle vivent les personnages, ou plutôt le sentiment d'insouciance de la petite fille. Quoi qu'il en soit, son statut d'adverbe de temps reste imprécis.

Il y a donc chez Rosa, comme chez Perrault, un petit pot, mais celui-ci contient des fruits au sirop à la place du beurre. Et pas de galette, ni aucun autre aliment : le panier est quasi vide car la petite fille va cueillir des framboises. En passant par le bois, elle ne voit que les bûcherons : pas de loup, ni réel ni métaphorique, car les bûcherons l'ont déjà exterminé. En l'absence du loup, plus de conversation possible dans le bois, alors c'est avec elle-même que la petite fille converse : "Je vais chez mémé, avec le panier et le pot, et le ruban vert dans les cheveux, ainsi que me l'a ordonné maman" ("Vou à vovó, com cesto e pote, e a fita verde no cabelo, o tanto que a mamãe me mandou»). Comme chez Perrault, la grand-mère habite après le moulin, mais il s'agit ici d'un moulin "qu'on pense voir» ("que a gente pensa que vê»). Par ailleurs c'est elle qui décide de prendre le chemin le plus long et elle apprécie tout ce qu'elle rencontre.

En arrivant chez sa grand-mère, elle frappe à la porte et entend : "Tire la chevillette en bois de la porte, entre et ouvre. Que Dieu te bénisse» ("Puxa o ferrolho de pau da porta, entra e abre. Deus te abençoe»). Cette simple phrase comporte une inversion : «entre et ouvre». Une fois encore, on assiste à un déplacement sémantique qui ébranle un peu le sens logique, car bien évidemment on ne peut pas entrer sans avoir d'abord ouvert la porte et, après qu'on est entré, "ouvrir» n'aura de sens que s'il reçoit un nouveau complément. Or aucun n'est disponible dans la phrase et on se voit à nouveau confronté à un "trou " syntaxico-sémantique, qui invite le lecteur à donner sens au verbe «ouvrir» en le complétant des éléments qu'il jugera pertinents.

La petite fille obéit à sa grand-mère et entre chez elle. La vieille dame, très faible, l'invite à la rejoindre «pendant qu'il est encore temps» («enquantoé tempo»). La tristesse s'installe et, à ce moment, la petite fille se rend compte qu'elle a perdu le ruban vert qu'elle portait dans les cheveux. Cette perte anticipe un peu sur le sentiment qui s'imposera à la fin du conto et elle 
renvoie à l'anticipation initiale : la petite fille avait été présentée comme «celle qui pour le moment». On sent qu'est venu ce moment annoncé au premier paragraphe. Et le dialogue commence entre deux femmes, le loup étant absent de l'histoire. Cependant, le sentiment de peur s'amplifie et la proximité d'un événement terrible opère une identification immédiate du lecteur à la petite fille, dans la mesure où il peut parfaitement reconnaître le type de peur qui terrifie Fita-Verde.

Au cours du dialogue, celle-ci remarque la maigreur extrême des bras de sa grand-mère, ses mains qui tremblent. Et la vieille femme explique : "C'est parce que je ne pourrai plus jamais te serrer dans mes bras, ma petite...» ("É porque não vou poder nunca mais te abraçar, minha neta...»). Ensuite, la petite fille observe les lèvres violacées de la vieille, qui dit : «C’ est parce que je ne pourrai plus jamais t'embrasser, ma petite" ("É porque não vou nunca mais poder te beijar, minha neta...»). Et, finalement, elle remarque les yeux malades et immobiles de la vieille, dans son visage très pâle. Celle-ci explique encore : «C'est parce que je ne te vois déjà plus, jamais plus, ma petite» (« $E$ porque já não estou te vendo, nunca mais, minha netinha...»). Le dialogue revêt un intérêt supplémentaire, en raison du choix sémantique opéré par l'auteur. À chaque réplique de la grand-mère, un verbe introductif est utilisé, formant une gradation toujours plus proche de la mort : «la grand-mère murmura» («a avó murmurou»), «la grand-mère soupira» («a avó suspirou»), "la grand-mère gémit» ("a avó gemeu »). À la fin, au moment où Fita Verde allait être sage pour la première fois, précise le narrateur, elle pousse un cri : "Mémé, j’ai peur du Loup!..." ("Vovozinha, eu tenho medo do Lobo!...»). Un loup qui revient avec la majuscule, loup plus terrifiant que jamais, car il n'est pas une menace animale visible, mais bien la mort elle-même.

Ce conto est très inquiétant dès la première lecture, et n'offre pas la fin heureuse et confortable du texte des frères Grimm. Au contraire, ici la mort est victorieuse, imprimant en la petite fille ce sentiment de finitude qui hante chaque existence. Ce trait, beaucoup plus proche du texte de Perrault que de celui des Grimm, complique l'accès de ce conto à un enfant. Tant sur le plan linguistique, car il n'est pas facile à lire (ni à traduire), que sur le plan des idées qu'il véhicule, Fita verde no cabelo ne paraît pas assimilable à n’importe quel âge. Ce conto a pourtant été édité séparément, accompagné d'illustrations qui le banalisent, comme s'il s'agissait d'une œuvre pour la jeunesse. C'est d'ailleurs ce qui est déclaré dans sa présentation : «Ce livre est aujourd'hui consacré comme un classique de notre littérature d'enfance et de jeunesse ${ }^{26}$.» Bien évidemment, de jeunes lecteurs peuvent lire Fita

26. J. G. Rosa, Fita verde no cabelo, I992, p. 34. 
verde no cabelo, mais un travail d'accompagnement est nécessaire pour que cette lecture leur soit profitable.

L'édition séparée du conto ne délivre pas toutes les informations sur ses éditions précédentes. On peut y lire que le conto a été extrait de Ave, palavra, recueil posthume composé par l'auteur. Ce livre est le dernier à figurer dans la chronologie de ses œuvres complètes. L'auteur indique qu'il $\mathrm{y}$ « réunit des notes de voyage, journaux, poésies, contos, petites chroniques, reportages poétiques et méditations, tout ce qui constitue sa collaboration de vingt ans, de façon discontinue et irrégulière, dans des journaux et revues brésiliens, pendant la période de 1947 à $1967^{27}$ ». Ce que l'édition séparée n'indique pas et que l'on peut lire dans l'édition des ouvres complètes, c'est la date de la première publication du texte, dans le journal $O$ Estado de S. Paulo : le 8 février 1964.

Cet indice, jamais exploité par la critique, donne une autre nuance à la peur thématisée dans le conto. Comme tout le monde le sait, I964 est l'année du coup d'état militaire au Brésil, qui s'est produit au mois de mars. C'est-à-dire qu'au moment de sa publication, Fita verde no cabelo a circulé dans une société préoccupée par les manifestations anti-communistes et contre le gouvernement de João Goulart, qui proposait des réformes assez progressistes et beaucoup plus proches du socialisme que de l'idéologie répressive de la droite de l'époque. Des mois durant la société civile a organisé des manifestations, finissant par appuyer le coup d'état qu'on a alors appelé «révolution». C'est bien une période d'inquiétude et de peur, une peur que les vingt ans qui ont suivi ont largement consolidée.

Nous précèderons dans une autre étude à l'analyse du journal qui publia pour la première fois Fita verde no cabelo et l'on pourra alors vérifier comment se construisit la co-textualisation et même la contextualisation du conto lors de sa première lecture par le public brésilien, suivant l'usage que font de ces concepts Heidmann et Adam, en faveur de la lecture dialogique de textes qui, eux aussi, se répondent dialogiquement :

Le texte qui répond à un autre crée des effets de sens nouveaux et radicalement différents.

Dans cette optique, un conte construit ses effets de sens en réponse à d'autres contes et à d'autres types de textes et de discours, dans un jeu perpétuel de différenciation, de variation et de renouvellement de sens inhérent au procédé du dialogue intertextuel ${ }^{28}$.

C'est valable pour chaque texte au moment de sa publication, car il s'insère dans un mouvement culturel, discursif et même politique agissant

27. J. G. Rosa, Ave, Palavra, 1994, p. 916.

28. U. Heidmann, Textualité et intertextualité des contes, 20I0, p. 37. 
sur sa réception de la même façon qu'en amont, un mouvement du même ordre avait d'abord agi sur sa production. Ainsi, chaque conto et le poème présentés ici mériteraient aussi une lecture de leur co-textualisation qui ne sera pourtant pas présentée ici. Toutefois, l'insertion de Fita verde no cabelo dans Ave, palavra, recueil posthume organisé par l'auteur, comme on l'a expliqué précédemment, offre déjà une brève appréciation de sa co-textualisation.

Dans Fita verde no cabelo, l'absence du loup interdit évidemment la reproduction de la scène où celui-ci avale grand-mère et petite fille. Pourtant, dans un autre conto, O porco e seu espírito (Le porc et son esprit), placé immédiatement avant Fita verde no cabelo, Rosa invente une situation intéressante. Deux voisins, Migudonho et Teixeirete, ne sont pas de vrais amis et vivent en entretenant certains soupçons sur l'autre, surtout Migudonho. Celui-ci possède un porc énorme, que le voisin lui a conseillé de vendre vivant, mais, pour contredire Teixeirete, il préfère tuer l'animal, comme on le faisait dans la campagne, au Brésil. Au lieu d'en vendre la viande, il décide de tout manger, et ordonne à sa femme de préparer immédiatement tous les morceaux. Son voisin est spectateur de la scène, en compagnie de sa famille, ce qui fait plaisir à Migudonho, qui se prend pour Hercule en train de manger devant un voisin en lui faisant envie.

Dans $O$ porco e seu espirito, Migudonho est victime d'un terrible mal au ventre, qui l'empêche de manger davantage. Et il en vient à souffrir atrocement, comme si l'opération s'était inversée et qu'il était attaqué de l'intérieur par le porc déjà avalé. Comme le dit le texte : «Le porc devient le sujet, et non l'objet de l'actuelle représentation» ("O porco fazia-se o sujeito, não o objeto da atual representação»).

Tout en ayant l'apparence d'une petite chronique rurale, ce conto renvoie à celui qui le suit dans l'organisation du recueil, car il présente la scène qui a été omise dans l'autre. Le porc est perçu comme vivant dans le ventre de Migudonho, exactement comme la grand-mère et la petite fille dans le ventre du loup : «Mais Migudonho n'était plus seulement Migudonho. Ils avaient mal, lui et le porc, tellement ils étaient unis, inséparables, irréductibles.» ("Mas Migudonho náo era mais só Migudonho. Doíam, ele e o porco, tão unidos, inseparáveis, intratáveis. "). Il faut encore dire que le titre de ce conto : Le porc et son esprit ( $O$ porco e seu espirito), joue avec l'expression brésilienne "esprit de porc» ("espirito de porco»), utilisée pour désigner les personnes dotées d'un esprit malin.

Une autre scène absente de Fita verde no cabelo, celle du dialogue entre la petite fille et le loup dans la forêt, qui caractérise la séduction, dans le sens étymologique du mot (sortir du chemin), peut trouver son reflet dans 
le texte qui lui succède au sein du recueil. Il s'agit d'un texte hybride sur le plan générique, car sous le titre Du journal à Paris (Do diário em Paris), il réunit quelques notes de journal intime, des petits poèmes et mêmes des aphorismes, qu'il organise en deux parties : la première a été écrite entre mai I953 et avril 1954, et la deuxième, en août 1949. La première nous intéresse un peu plus que l'autre, car elle paraît répondre à Fita verde no cabelo : elle relate la rencontre entre l'écrivain et une dame grecque, qu'il voit plusieurs fois à Paris, et qui occupe l'essentiel du texte, faisant l'objet de poèmes et de dialogues très séducteurs, parfois explicites. Parmi les fragments qui composent ce texte, celui qui a été placé avant l'apparition de la "fille grecque» ("moça grega», qu'il appelle "fille» à cause de son âge apparent, mais qui en fait est mariée) est particulièrement intéressant. Il s'agit justement d'un fragment qui recourt à la couleur à laquelle il renoncera, dix ans plus tard, pour raconter à sa façon l'«estória» du Chaperon Rouge :

No metrô, em vermelho, este anúncio, que é Paris e é um poema:

"le

rouge baiser

permet

le baiser..." 29

Dans le métro, en rouge, cette annonce que c'est Paris et c'est un poème :

"le

rouge baiser

permet

le baiser...”

Le dialogue entre les textes s'impose d'autant plus que l'organisation du livre relève de l'auteur lui même; et elle n'obéit pas un simple critère chronologique ni même à un critère de classement par journaux et revues les ayant publiés. Quoi qu'il en soit, O porco e seu espirito et Du journal à Paris répondent très bien, comme on l'a montré, à des éléments, notamment manquants, de l'histoire de Fita Verde.

Les quatre réécritures du Petit Chaperon Rouge présentées dans cet article ont été publiées au Brésil dans la période du régime militaire : Fita verde no cabelo, de G. Rosa, en 1964, l'année du coup d'état; Chapeuzinho Vermelho, de Millôr Fernandes, en 1967; Chapeuzinho vermelho de raiva, de Mário Prata, en 1970; et Chapeuzinho Amarelo, de Chico Buarque, en 1979. Ce

29. J. G. Rosa, Ave, Palavra, 1994, p. 984.

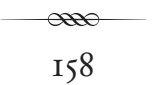


corpus s'ouvre et s'achève sur la peur : instauration de la peur chez Rosa et méthode pour s'en débarrasser chez Buarque. Millôr et Prata nous offrent plutôt la recette pour vivre avec elle : l'humour.

L'autre fait intéressant, si l'on considère la période en question, est le changement de la couleur couvrant la tête de la petite fille chez Rosa et Buarque. Tous les deux ont choisi des couleurs qui ont une forte connotation nationaliste, étant donné que ces deux couleurs, le vert et le jaune, présentes dans le drapeau brésilien, sont aussi les couleurs officielles emblématiques du pays. De nouveaux éléments permettront bientôt une relecture du conto de Rosa, tenant compte de sa contextualisation. En plus de la date révélatrice de sa première publication, la lecture du journal où il a paru permettra peut-être de découvrir les implications politiques de ce conto, quoique Rosa ait dit un jour :

Embora eu veja o escritor como um homem que assume uma grande responsabilidade, creio entretanto, que não deveria se ocupar de política; não desta forma de política. Sua missão é muito mais importante: é o próprio homem ${ }^{30}$.

Tout en considérant l'écrivain comme un homme qui a une grande responsabilité, je crois, cependant, qu'il ne devrait pas s'occuper de politique; au moins pas de cette forme de politique. Sa mission est beaucoup plus importante : c'est proprement l'homme.

Si c'est de l'homme qu'il s'agit, on parle alors de la vraie politique. Et si Fita verde no cabelo est dépourvue d'une quelconque intention politique de son auteur, du fait d'avoir été mis en circulation pendant l'une des périodes les plus difficiles de l'histoire brésilienne, c'est un conto politique, qui permet de penser le problème le plus grave de chaque être humain : sa propre fin - loup qui garde en soi toute la perversité possible.

\section{Bibliographie}

Buarque Chico, Chapeuzinho Amarelo, Rio de Janeiro, José Olympio, 2005 [1979].

Fernandes Millôr, Liçôes de um ignorante, Rio de Janeiro, José Álvaro Editor, 1967.

Grimm Jacob et Wilhelm, Contes pour les enfants et la maison, édités et traduits par Natacha Rimasson-Fertin, coll. «Merveilleux», nº 40, tome I, Paris, José Corti, 2009.

30. G. Lorenz, «Diálogo com Guimarães Rosa», 199I, p. 62-63. 
Heidmann Ute, «Expérimentation générique et dialogisme intertextuel : Perrault, La Fontaine, Apulée, Straparola, Basile», Féeries, nº 8, 20II, p. 45-69.

Heidmann Ute et Adam Jean-Michel, Textualité et intertextualité des contes - Perrault, Apulée, La Fontaine, Lhéritier..., Paris, Classiques Garnier, coll. «Lire le XVII ${ }^{\mathrm{e}}$ siècle», vol. 2, 2010.

Lorenz Günther, "Diálogo com Guimarães Rosa», dans A. Coutinho (dir.), Guimaráes Rosa, Rio de Janeiro, Civilização Brasileira, I99I, coll. "Fortuna Crítica», vol. 6. Selection de textes par Eduardo F. Coutinho. Meireles Cecília, Problemas da literatura infantil, Rio de Janeiro, Nova Fronteira, 1984, p. 37-38.

Monteiro Lobato José Bento, O Picapau Amarelo, São Paulo, Brasiliense, I971, p. 55-57.

Monteiro Lobato José Bento, Contos de Grimm, traduits et adaptés, São Paulo, Brasiliense, I969.

Monteiro Lobato José Bento, Novos contos de Grimm, traduits et adaptés, São Paulo, Brasiliense, 1969.

Perrault Charles, Contos e fábulas, traduit par Mario Laranjeira, São Paulo, Iluminuras, 2007.

Perrault Charles, Grimm Jacob et Wilhelm et Andersen Hans Christian, Contos de fadas, traduit par Maria Luiza X. de A. Borges et présentés par Ana Maria Machado, Rio de Janeiro, Zahar, 20Io..

Prata Mario, Chapeuzinho vermelho de raiva, Rio de Janeiro, Globo, 1979. Rosa João Guimarães, Ave, Palavra [1970], dans João Guimarães Rosa Ficção completa, vol. 2, Rio de Janeiro, Nova Aguilar, I994.

Rosa João Guimarães, Fita verde no cabelo - nova velha estória, illustr. par Roger Mello, Rio de Janeiro, Nova Fronteira, 1992.

Volobuef Karin, "Comentário sobre as traduçóes brasileiras dos irmãos Grimm ». Disponible sur <http://volobuef.tripod.com/index. html\#Conto\%2ode\%2ofadas> [consulté le I4 mai 20I2]. 